system can display the heuristic models it has acquired in intelligible and transmissible symbolic forms. Rules induced from example data should approximate to what humans would call concept descriptions.

The rule-induction approach to empirical learning, pioneered by R.S. Michalski at Illinois and by J.R. Quinlan in Sydney, is well represented in Machine Learning. Beyond heuristic know-how, automated acquisition from data of causal and descriptive models can be made to lead from machine learning to machine discovery. It is pleasing that the great founder of this school, Herbert Simon, is a contributor of new work to the journal's first issue. Most gratifying of all is the evidence of progress in readying machine learning for its coming role in scientific investigation for which it must itself become more scientific. In a guest editorial to a notable special issue of Machine Learning (Vol. 2, No. 4, April 1988), David Haussler remarks that "there is an art to designing efficient and effective learning algorithms. Too much of an art. Many researchers have argued that if the field is ever to fully mature, we must find a sound and adequate basis on which to build a science of machine learning".

That the building proceeds apace can in part be judged from papers in this landmark number of Machine Learning. On the experimental side, a surge of even more recent publication attests to the quickening tempo. I would prescribe at least a glance through the 50 or so communications to the Fifth International Conference on Machine Learning just published by Morgan Kaufmann. The 'helps for the mind' theme is evident, as in the communication by Cheeseman and colleagues of machine discovery from raw stellar spectral data of new categorizations of stars. These categorizations were new to the NASA astronomers and recognizably of clear physical significance.

In envisaging the future edifice of scientific knowledge, Francis Bacon knew that it could only be constructed at whatever pace might be permitted by advances in instrumentation. In modern times the term instrumentation is extending into the burgeoning world of scientific software. But Bacon also saw that ultimately instrumentation of the intellectual process itself would hold the key. His list, appended to his Advancement of Learning of the "deficiencies of knowledge . . . to be supplied by posterity" contains the item "The True Use of Induction in Philosophy" followed closely by "A New Engine; or helps for the mind corresponding to those of the hand". Bacon's motivation was practical. Four hundred years later we are seeing the first conversions of practicality into practicability.

Donald Michie is Chief Scientist of the Turing Institute, George House, 36 North Hanover Street, Glasgow G1 $2 A D$, UK.

\section{Tapping into three dimensions}

\author{
William C. Ripka
}

Journal of Computer-Aided Molecular Design. Editors-in-chief G.R. Marshall, J.G. Vinter and H.-D. Höltje. ESCOM, PO Box 214, 2300 AE Leiden, The Netherlands. 4/yr. Dfl 380, $\$ 190$.

THE phrase 'computer-aided drug design' is becoming one of the most abused in the English language. Although it is to be seen in the titles of several books and journal articles, one is often hard pressed to find any further mention of the design of any molecules beyond the words in the title. In fact, this is a legitimate and promising arena of research, which may provide the necessary link between the findings of molecular biology and conversion of this knowledge of macromolecules into the production of smaller, more manageable molecules.

Articles that have actually dealt squarely with the subject have been spread among an assortment of journals primarily serving other subjects (biology, physical chemistry, chemical synthesis, computer graphics, protein structure and so on). The object of the Journal of Computer-Aided Molecular Design is to rectify this unsatisfactory situation by bringing together reports of research directed towards all of the applications implied by its title. The editors state that the purpose of their journal is threefold to be a forum for multidisciplinary communication, in recognition of the several areas that molecular design encompasses (computer science, chemistry, biology and structure-activity relationships); to provide a source of references to aid researchers in keeping abreast of developments; and (interestingly) to catalyse the use of electronic publishing by including an electronic version of the journal which "will enable the 'reader' to view the figures on his own terminal in three dimensions".

With respect to the first goal, the editors have been remarkably successful. The quality of the contributions has been consistently high in the first year of publication, and the papers have indeed dealt with a wide range of topics - theoretical and computational chemistry, computer graphics, drug design and molecular modelling, associated techniques in protein engineering and structure-activity relationships in general. One possible pitfall in attempting to cover such a broad scope is that the prime intention, namely, to deal with molecular design, is lost sight of. Yet the editors have managed to keep the emphasis of most of the contributions, whether theoretical or applied, firmly on the design problem.

In addition to research contributions, a "Perspective" section is included in which appear excellent short summaries of important new topics; those by van Gunsteren et al. (Vol. 1, July 1987) on free-energy perturbation and by DonneOp den Kelder (Vol. 1, Oct. 1987) on distance geometry analysis of ligandreceptor site binding are especially good. The overall quality of production is excellent, with crisp colour photographs of computer graphics being included.

One of the most admirable of the journal's goals is to provide machinereadable results (ASCII files on IBM or Macintosh disks) from the various molecular modelling and enzyme-binding studies reported in the articles. The availab-

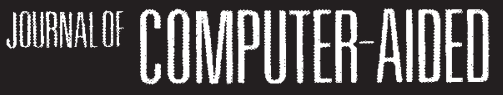 MOLECULAR DESCGON}

ility of coordinates from these investigations should allow access to the research in a much more usable form than the usual diagrams and two-dimensional views, and should encourage further refinements and studies of the models concerned. The availability of this machine-readable data from the first volume was announced in the April 1988 issue.

For both contributors and subscribers, Journal of Computer-Aided Molecular Design fills an important role. Further, by making the actual coordinates of the model studies available via ASCII files the journal is pioneering the communication techniques of the future. It should be considered essential reading for anyone interested in molecular modelling and molecular design.

William C. Ripka is Supervisor of the Molecular Modeling Group, Medical Products Department, E.I. DuPont De Nemours \& Company, Wilmington, Delaware 19898, USA.

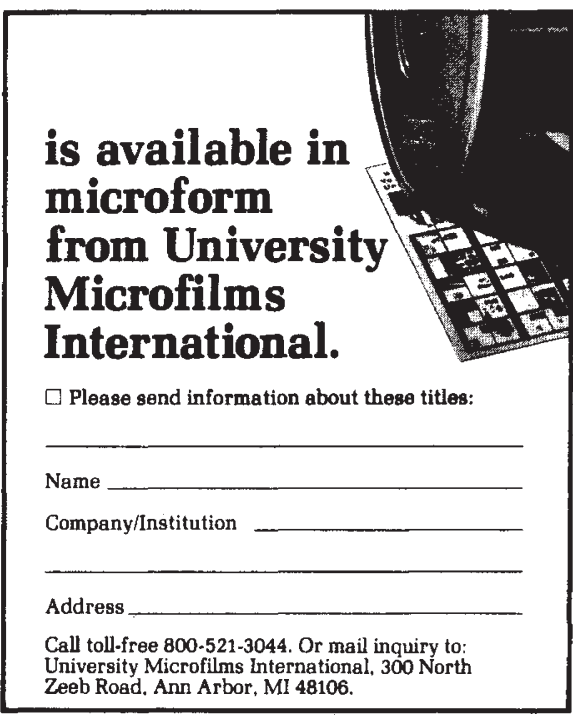

